\title{
The Molecular Structure of Biphenyl and some of its Derivatives
}

\author{
O. B A S T I A N S EN
}

Universitetets Kjemiske Institutt, Blindern - Oslo, Norway

$\mathbf{I}_{\mathrm{t}}^{\mathrm{t}}$

t has been known for a long time that atrop-isomerism will occur when

two or more of the ortho hydrogen atoms in biphenyl are replaced by larger atoms or groups. The phenomenon is easily explained as due to steric hindrance which prevents rotation about the central $\mathrm{C}-\mathrm{C}^{\prime}$-bond in the biphenyl derivative. For instance Searle and Adams ${ }^{1}$ have separated 2,2'-diiodo-4,4'dicarboxybiphenyl and the corresponding bromocompound in $d$ - and $l$-components. These molecules must consequently have a non-planar configuration. On the other hand X-ray crystallographic investigations have shown that meta- and para-substituted biphenyls as well as biphenyl itself have a completely planar configuration ${ }^{2-5}$. This fact has been explained by Sutton ${ }^{6}$ and others ${ }^{7}$ as a result of the partial double-bond character of the central C-C $\mathrm{C}^{\prime}$-bond.

The ultraviolet spectroscopists ${ }^{8}$ have studied many biphenyl derivatives and have found a marked difference in the ultraviolet absorption of the orthosubstituted and the non-ortho-substituted derivatives. Their view is that the ortho-substituted derivatives have a non-planar configuration and the others a completely planar configuration. They expect this to be found true not only in the crystalline state but also in liquids and gases.

The dipole moment measurements do not seem to be able to give information about the angle between the ring planes in the biphenyl derivatives. As an example of the numerous dipole moment measurements of these derivatives we may mention the series $4,4^{\prime}-, 3,3^{\prime}$-, and $2,2^{\prime}$-dichlorobiphenyl ${ }^{12}$, 13 which have been measured in solution. As was to be expected, the first of these has zero dipole moment. This shows that the atoms $\mathrm{Cl}_{4}, \mathrm{C}_{4}, \mathrm{C}_{1}, \mathrm{C}_{1}{ }^{\prime}, \mathrm{C}_{4}{ }^{\prime}$ and $\mathrm{Cl}_{4}{ }^{\prime}$ lie on a straight line. The other two compounds have dipole moments $1,68 \mathrm{D}$ and 1,77D respectively. The plane trans form can therefore be excluded in 


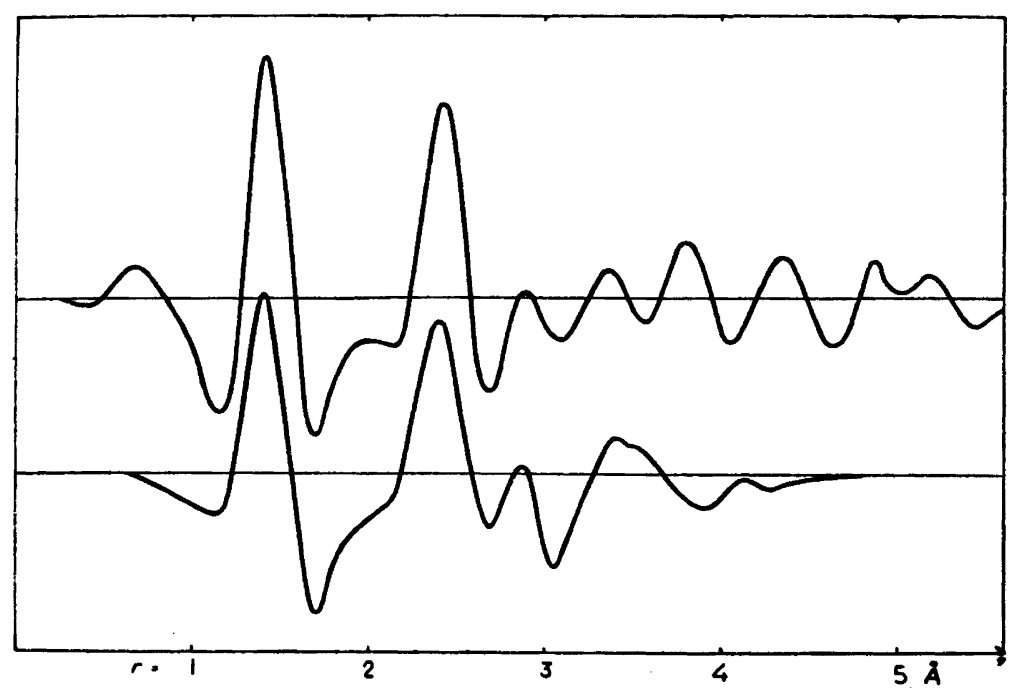

Fig. 1. $\sigma(r)$-curve for biphenyl and benzene.

both these cases, but we have to make a choice between three possibilities: 1) An equilibrium between cis and trans forms. 2) A free or restricted rotation about the central $\mathrm{C}-\mathrm{C}^{\prime}$-bond. 3) A rigid non-planar configuration.

Karle and Brockway ${ }^{14}$ have investigated gaseous biphenyl by the visual electron diffraction method. Their results do not seem to be entirely free from ambiguity, but they think they have found indications of a non-planar model.

We have by the electron diffraction sector method studied the structure of biphenyl, 3,3'-dichlorobenzidine, and 3,3'-dibromobiphenyl.

\section{RESULTS AND DISCUSSION}

Biphenyl. In Fig. 1 the upper curve is the $\sigma(r)$-curve for biphenyl. The lower curve is the $\sigma(r)$-curve for benzene multiplied by 2 . The peak corresponding to the $\mathrm{C}-\mathrm{C}$-bond distances in the rings occurs at $1.40 \AA$. If there exist different $\mathrm{C}-\mathrm{C}$-bond distances in the phenyl rings, this can not be detected from the $\sigma(r)$-curve. The two curves are for the interval $0>r>3 \AA$ similar in their broad features. This is in accordance with the assumption that the phenyl rings in biphenyl to the first approximation are indentical with the benzene ring. The differential curve should therefore for the most part contain peaks corresponding to atomic distances between the two rings in biphenyl. The differential $\sigma(r)$-curve is given as the upper curve, A, in Fig. 2. The curves B, C, and D are theoretical differential curves calcu- 


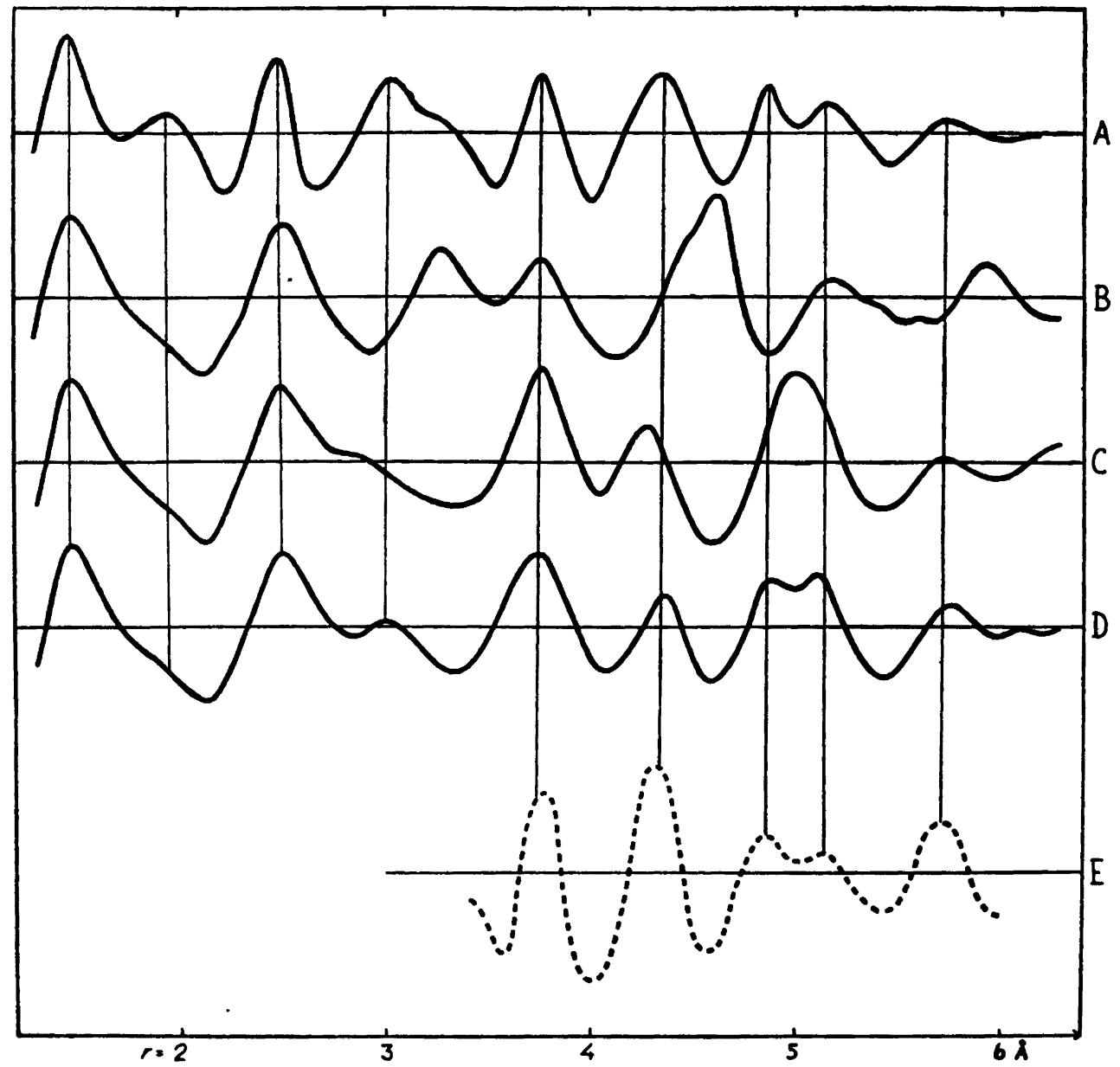

Fig. 2. Experimental and theoretical differential $\sigma(r)$-curves. $\left(\sigma(r)_{\text {biphenyl }} \div 2 \cdot \sigma(r)_{\text {benzene }}\right)$.

lated on different assumptions as regards the angle $\varphi$ between the ring planes. The $\varphi$-values are $90^{\circ}, 0^{\circ}$, and $45^{\circ}$ for the curves $\mathrm{B}, \mathrm{C}$, and $\mathrm{D}$ respectively. Some of the peaks in the experimental curve are found in all the theoretical curves. The corresponding distances are those which do not vary when the two rings are rotated relatively to each other. These peaks will indirectly give informations of the length of the central $\mathrm{C}-\mathrm{C}^{\prime}$-bond which is found to be $1.48 \mathrm{~A}$.

The theoretical curve which is in best accord with the experimental one is undoubtedly curve $D$. Though the similarity of the two curves $A$ and $D$ is striking, especially with regard to the position of the maxima, a real deviation 


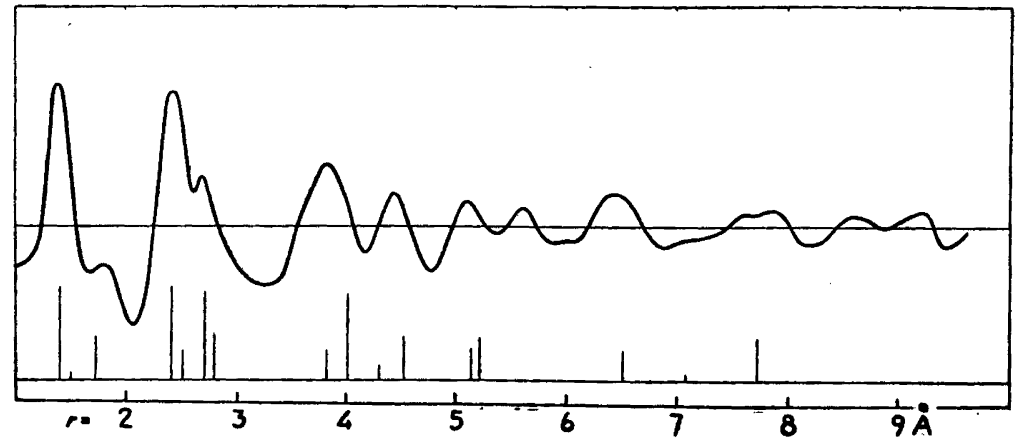

Fig. 3. $\sigma(r)$-curve for 3,3'-dichlorobenzidine.

is observable, particularly for the interval $1>\mathrm{r}>3 \AA$. The $\sigma(r)$-curves for biphenyl and benzene have in that interval the highest maxima and the deepest minima, and errors will therefore influence the differential curve to a relatively greater extent. The lower curve $\mathrm{E}$ in Fig. 2 is an experimental differential curve calculated from a new series of diagrams. This reinvestigation was done about a year after the calculation of the curves $A, B, C$, and D. It was carried out in order to demonstrate the reproducibility of the method in this particular case. The curve $\mathrm{E}$ was calculated only for the interval which is important for our studies.

From these considerations it seems evident that we should be able to exclude both the model which has the two ring planes perpendicular to each other and the strictly planar model found in the crystalline state. An intermediate configuration must therefore be assumed, but in spite of the rather good agreement between experiments and theory for the $45^{\circ}$ model, it is very difficult by a systematic variation of the angle to settle the limits of the error. A rough estimation suggests, however, that the deviation from the $45^{\circ}$ value is less than $\pm 10^{\circ}$. - In this connection we may mention that an exclusion of minor oscillations about the equilibrium state is impossible from our $\sigma(r)$ curves.

3,3'-Dichlorobenzidine. Fig. 3 shows the experimental $\sigma(r)$-curve for $3, \mathbf{3}^{\prime}$ dichlorobenzidine. Most of the peaks occurring in the $\sigma(r)$-curve can be explained by distances which do not vary by a rotation about the central C-C'bond. This is demonstrated in a simple manner by the line diagram below the $\sigma(r)$-curve. (The $\mathrm{C}-\mathrm{C}$ - and the $\mathrm{C}-\mathrm{Cl}$-bond distances are found to be $1.40 \AA$ and $1.73 \AA$ respectively). Of the remaining peaks the most pronounced one occurs at $5.6 \AA$. It is readily interpreted as a $\mathrm{C}_{2}-\mathrm{Cl}^{\prime}$-distance, if the angle between the two ring planes is assumed to be approximately $52^{\circ}$. The rest of 
the $\sigma(r)$-curve does not seem to give further reliable information concerning the value of the angle. On the other hand a planar model can not by any means be compatible with the $\sigma(r)$-curve.

3,3'-Dibromobiphenyl. This compound must be expected to be very well suited for the study of our effects. The bromine substituted hydrocarbons have in many cases proved to be better fitted for electron diffraction than the derivatives containing other halogens. The $3,3^{\prime}$-dibromobiphenyl possesses further the advantage over the $3,3^{\prime}$-dichlorobenzidine of the absence of the amino groups.

The $\sigma(r)$-curve for 3,3'-dibromobiphenyl is given in Fig. 4. All the maxima for $r<5.5 \AA$ may easily be explained by distances which do not vary when the two ring planes are rotated relatively to each other. These distances are indicated by the solid line diagram below the $\sigma(r)$-curve. The bond distances used in the calculation of the line diagram are: $\mathrm{C}-\mathrm{C}=1.40 \AA, \mathrm{C}-\mathrm{C}^{\prime}=$ $1.49 \AA$ and $\mathrm{C}-\mathrm{Br}=1.88 \AA$. The maximum at $3.4 \AA$ which does not correspond to any internuclear distance may easily be explained as a diffraction error maximum caused by the neighbourhood of the peaks at $2.86 \AA$ and 4.18 $\AA$. Besides the peaks mentioned which are easily interpretable a further six peaks, which must be explained as internuclear distances, have been observed.

Table 1. Values characterizing the magnitude of the deviation from coplanarity of $3,3^{\prime}$. dibromobiphenyl.

$\begin{array}{ccc}\begin{array}{c}\text { The } r \text {-values of } \\ \text { the observed } \\ \text { maxima }\end{array} & \text { Distances } & \varphi \\ 5.80 \AA & & \\ 6.49 & \mathrm{Br}_{3}-\mathrm{C}_{6}{ }^{\prime} & \mathbf{5 6}^{\circ} \\ 7.14 & \mathrm{Br}_{3}-\mathrm{C}_{2}{ }^{\prime} & 4^{\circ} \\ 7.65 & \mathrm{Br}_{3}-\mathrm{C}_{5}^{\prime} & 62^{\circ} \\ 8.07 & \mathrm{Br}_{3}-\mathrm{C}_{3}{ }^{\prime} & 5^{\circ} \\ 9.20 & \mathrm{Br}-\mathrm{Br}_{\mathrm{c}} & 5^{\circ} \\ & \mathrm{Br}-\mathrm{Br}_{\mathrm{t}} & 5^{\circ}\end{array}$

mean value $54^{\circ}$

The position of these peaks (dashed line in the line diagram) is shown in the first column of Table 1. They correspond to distances occurring in a nonplanar structure (Second column of Table 1). These distances belong to such as vary when the two ring planes are rotated relatively to each other. The other distances of this type will be unable to influence the $\sigma(r)$-curve to any appreciable extent. The distances $\mathrm{Br}-\mathrm{Br}_{\mathrm{c}}$ and $\mathrm{Br}-\mathrm{Br}_{\mathrm{t}}$ from second column in Table 1 are the two different $\mathrm{Br}-\mathrm{Br}$-distances which may occur in a molecule when the two ring planes are not at right angles to each other. The values 


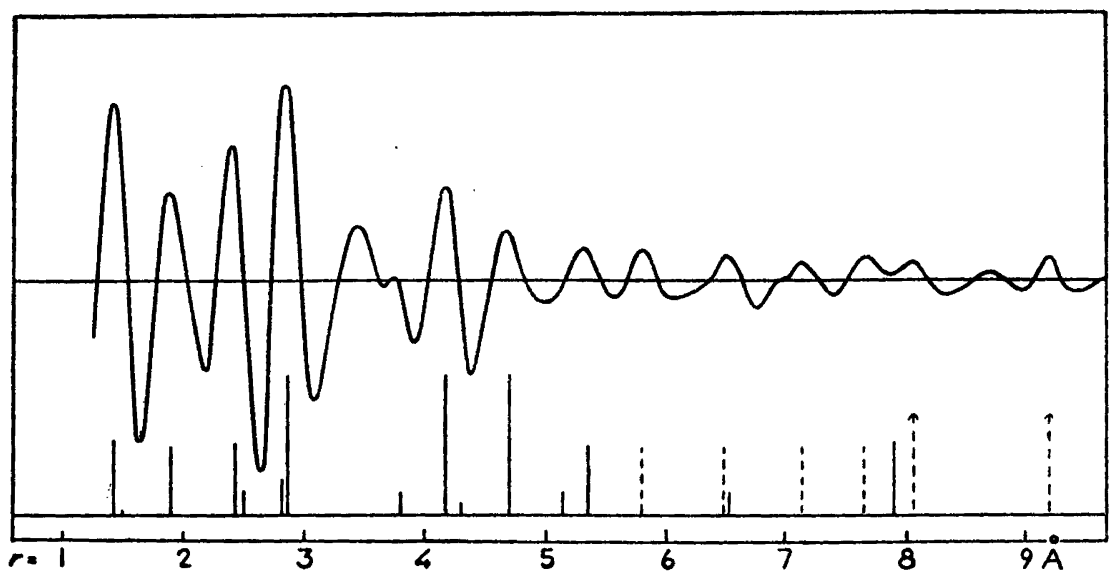

Fig. 4. $\sigma(r)$-curve for 3,3'-dibromobiphenyl.

in the first column of Table 1 enable us to calculate six independent values for the angle $\varphi$ between the ring planes. These values are given in the third column of Table 1. Though there exist some fluctuations in the $\varphi$-values thus obtained, the effect of the deviation from coplanarity seems to be demonstrated in a conclusive manner. The error in the determination of the angle $\varphi$ is presumabely less than $5^{\circ}$, and in this case therefore we can give a real quantitative determination of the effect.

The deviation in the values of the angle $\varphi$ for the three compounds under consideration is within the limits of the error. The non-planar configuration must in all the three cases be explained by the repulsive forces between adjacent hydrogen atoms. Dipole effects in $3,3^{\prime}$-dichlorobenzidine and $3,3^{\prime}$ dibromobiphenyl, might have been expected to contribute somewhat to the formation of the molecule configuration. The fact that these molecules have a very similar configuration to the biphenyl molecule shows that the intramolecular van der Waals forces certainly must have a predominant signification. The shortest $\mathrm{H}_{2}-\mathrm{H}_{2}{ }^{\prime}$-distances in a molecule with a $\varphi$-value of $54^{\circ}$ is about 2.65 $\AA$, corresponding to a van der Waals distance somewhat greater than that usually observed.

The usual assumption that resonance phenomena alone are responsible for the coplanarity of the biphenyl molecule and many of its derivatives in the crystalline state, is from our considerations obviously wrong. Of course resonance phenomena might contribute to the stabilization of the planar configuration in the crystalline state, but additional effects must be present. In a crystal not only intra-molecular forces will determine the shape of the 
molecule, the inter-molecular forces must also be expected to play a certain part. Though the energy of an isolated molecule may have a minimum for the non-planar configuration, it may well be that a planar molecule is better suited as a unit for the construction of a stable lattice.

Finally we may mention that Merkel and Wiegand ${ }^{15}$ in a recent work have independently come to results in good agreement with those given above. They find by ultraviolet spectroscopy that the molecules of biphenyl are planar in the crystalline state, but not in the liquid or gaseous state.

\section{SUMMARY}

The molecular structure of gaseous biphenyl, 3,3'-dichlorobenzidine, and $\mathbf{3}, \mathbf{3}^{\prime}$-dibromobiphenyl has been studied by the electron diffraction sector method. A non-planar configuration is found. The angle between the ring planes is found to be $45^{\circ} \pm 10^{\circ}, 52^{\circ} \pm 10^{\circ}, 54^{\circ} \pm 5^{\circ}$ for the biphenyl, $3,3^{\prime}$ dichlorobenzidine, and 3,3'-dibromobiphenyl respectively. The significance of the result is discussed.

I wish to express my gratitude to professor Dr. Roger Adams, University of Illinois for having placed a sample of 3,3'-dibromobiphenyl at my disposal. I also wish to thank Fridtjof Nansens Fond and Det Vitenskapelige Forskningstond av 1919 for economical aid without which this work could not have been accomplished.

\section{LITERATURE}

1. Searle, N. E., and Adams, R. J. Am. Chem. Soc. 55 (1933) 1649; 56 (1934) 2112.

2. Dahr, J. Indian J. Phys. 7 (1932) 43.

3. Toussaint, J. Acta Cryst. 1 (1948) 43.

4. Saunder, D. H. Proc. Roy. Soc. London A 188 (1946) 31.

5. Niekerk, J. N. van, and Saunder, D. H. Acta Cryst. 1 (1948) 44.

6. Sutton, L. E. Trans. Farad. Soc. 30 (1934) 791.

7. Pauling, L., and Sherman, J. J. Chem. Phys. 1 (1933) 679.

8. Pickett, L. W., Walter, G. F., and France, H. J. Am. Chem. Soc. 58 (1936) 2296.

9. Pestemer, M., and Mayer-Pitsch, E. Monatsh. 70 (1937) 104.

10. Friedel, R. A., Orchin, M., and Reggel, L. J. Am. Chem. Soc. 70 (1948) 199.

11. O'Shaughnessy, M. T., and Rodebush, W. H. J. Am. Chem. Soc. 62 (1940) 2906.

12. Hampson, G. C., and Weissberger, A. J. Am. Chem. Soc. 58 (1936) 2111.

13. Weissberger, A., Sängerwald R., and Hampson G. C. Trans. Farad. Soc. 30 (1948) 884.

14. Karle, J. L., and Brockway, L. O. J. Am. Chem. Soc. 66 (1944) 1974.

15. Merkel, E., and Wiegand, Ch. Z. Naturforsch. 3 b (1948) 93.

Received March 14, 1949 . 